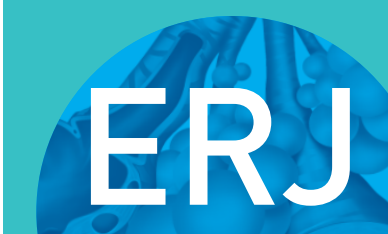

open research

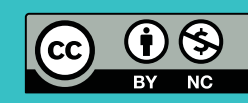

\title{
Influenza: from zoonosis to pandemic
}

\author{
Leslie A. Reperant ${ }^{1}$, Fleur M. Moesker ${ }^{2}$ and Albert D.M.E. Osterhaus ${ }^{1,3}$ \\ ${ }^{1}$ Artemis One Health Research Foundation, Utrecht, The Netherlands. ${ }^{2}$ Dept of Viroscience, Erasmus Medical \\ Centre, Rotterdam, The Netherlands. ${ }^{3}$ Research Centre for Emerging Infections and Zoonoses, University of \\ Veterinary Medicine, Hannover, Germany.
}

Correspondence: Albert D.M.E. Osterhaus, Research Centre for Emerging Infections and Zoonoses, University of Veterinary Medicine, Bünteweg 17, 30559 Hannover, Germany. E-mail: Albert.Osterhausatiho-hannover.de

@ERSpublications

Global surveillance and advances in vaccine technology are essential to answer the threat of influenza pandemics http://ow.ly/Yt3e4

Probable epidemic influenza outbreaks have been described as early as the 5th century BC, as part of the Cough of Perinthus associated with the winter solstice, in Hippocrates' Corpus Hippocraticum "Of the Epidemics" [1]. The word "influenza" was first introduced in the 16th century, defining the illness of the cold season that in the early 1930s was shown to be caused by "filterable agents", since then called influenza viruses. Three types or genera of influenza viruses have been recognised (influenza A, B and C viruses), while a fourth genus has been recently proposed [2]. Today, we distinguish three manifestations of influenza in humans, which may impose from mild to severe morbidity and mortality burdens: zoonotic, pandemic and seasonal influenza, all caused by an infection with an influenza virus. Although our arsenal of intervention strategies for influenza has advanced in the past decades and a growing public conception has arisen of influenza as a usually seasonal inconvenience, threats posed by influenza A viruses, with their different manifestations and associated complications, are continuously knocking on our door.

Morbidity and mortality burdens of influenza have affected humanity since ancient times. The most spectacular influenza burden is caused by pandemic influenza A viruses, upon their introduction in a human population with little or no pre-existing specific immunity. Four influenza pandemics, or global epidemics, have affected humanity in the past century. The most devastating of these occurred in 1918, causing influenza in about half the world population, with 30-50 million deaths worldwide, affecting principally the young and otherwise healthy [3]. The subsequent influenza pandemics of 1957 ("Asian flu”), 1968 ("Hong Kong flu") and 2009 ("Mexican flu") were milder, each claiming the lives of 0.3-2 million individuals. Pandemic influenza A viruses circle around the world in several waves, eventually replacing an existing seasonal influenza A virus. Seasonal influenza viruses cause annual winter epidemics that infect $5-15 \%$ of the world population, resulting in 3-5 million severe cases and 250000-500 000 deaths every year [4]. Seroprevalence studies reported that influenza viruses infect most children by the age of 6 years [5]. In fact, school-age children are considered primarily responsible for influenza virus transmission in the community [6]. Inter-pandemic periods have ranged between one and four decades, bringing collective seasonal influenza burdens to levels comparable to those reached by influenza pandemics.

The burden of zoonotic influenza A virus infections has made the headlines ever since the identification of avian and swine influenza viruses that, without apparent prior adaptation, infected and caused severe disease in humans and other mammals. The diversity of zoonotic influenza A viruses that circulate in avian or swine reservoirs expands every year. Influenza A viruses are classified into subtypes defined by their surface haemagglutinin and neuraminidase glycoproteins. Zoonotic influenza virus subtypes of particular concern include highly pathogenic avian influenza virus (HPAIV) H5N1, low pathogenic avian influenza viruses $\mathrm{H} 7 \mathrm{~N} 9, \mathrm{H} 9 \mathrm{~N} 2$ and most recently $\mathrm{H} 10 \mathrm{~N} 8$, as well as swine influenza viruses H1N1 and H3N2. Although, altogether, these viruses have caused disease or death in not more than 2000 individuals, their typically high case fatality rate (reaching, for example, up to $60 \%$ for hospitalised patients

Received: Feb 022016 | Accepted: Feb 072016

Conflict of interest: Disclosures can be found alongside this article at openres.ersjournals.com

Copyright $\odot$ ERS 2016. This article is open access and distributed under the terms of the Creative Commons Attribution Non-Commercial Licence 4.0. 
with an HPAIV H5N1 infection) is worrisome. Moreover, mortality caused by some of these viruses, including HPAIV H5N1, has been highest in children and young adults [7]. Zoonotic influenza viruses generally are little or not transmissible among humans via the air. Should they acquire such ability, they could spark a novel influenza pandemic.

Humankind is today better prepared to face a new influenza pandemic and to curb the burden of zoonotic, pandemic and seasonal influenza than ever before. However, continued effort is needed to fully build on our advanced knowledge of influenza threats and burdens in order to efficiently combat these infections. The recurring burden of seasonal influenza weighs heavier on the population of older adults and other at-risk groups, such as the very young, pregnant women and individuals with underlying conditions like immunocompromised individuals [4]. They may develop life-threatening complications requiring hospitalisation. These include the development of bacterial pneumonia and worsening of underlying conditions, but also primarily viral complications, such as viral pneumonia and influenza virus infection of the central nervous system [8]. In particular, paediatric patients aged $<5$ years are at high risk of developing severe disease, with a global incidence of influenza-associated severe acute lower respiratory tract infection estimated at 1 million cases, causing 28000-111500 deaths every year [9].

Timely administration of antivirals (currently in the form of neuraminidase inhibitors) is recommended and was recently shown to be beneficial in reducing severity and duration of influenza virus infection and preventing lower respiratory tract complications, thereby limiting hospitalisation and death [10], including in critically ill paediatric patients [11]. Moreover, the use of antiviral treatment in primary care is currently being studied $[12,13]$. The emergence of antiviral-resistant viruses nonetheless continues to threaten the efficacy of such treatments, especially in individuals with immunocompromised conditions [14]. This was for example illustrated in 2009 with the report of a 5-year-old boy with an underlying disease who was hospitalised with pandemic influenza (pH1N1) infection and succumbed despite treatment with oseltamivir, zanamivir and peramavir due to development of antiviral resistance [15].

Preventive influenza vaccines are by far the most cost-effective tools to combat seasonal influenza. Despite long-standing debates, they have been shown to be effective in preventing or reducing the severity of influenza and influenza-related disease and death in at-risk individuals [16]. The governments of many countries, such as the USA, Finland, the UK and Australia, have implemented vaccination programmes for healthy children of defined age groups to further limit transmission and spread of the viruses. Yet two major stumbling blocks impede the benefits of seasonal influenza vaccination: overall low vaccination coverage, and the need for continuous update of the influenza vaccine strains to match circulating strains evolving through antigenic drift. The use of alternative routes of administration of influenza virus vaccines, such as the intranasal application of live attenuated vaccines, especially in children, together with the advent of large meta-analyses demonstrating the benefits of influenza vaccination, may favour higher coverage rates in the future.

Seasonal influenza A viruses circulate throughout the year in East and Southeast Asia, which are considered the source regions of the $\mathrm{H} 3 \mathrm{~N} 2$ strains that cause winter epidemics in the northern and southern hemispheres [17]. A different pattern of global circulation of H1N1 viruses, with longer local persistence and less frequent global movement, has recently been described [18]. Seasonal influenza H3N2 and, to a lesser extent, H1N1 strains constantly evolve to escape pre-existing immunity in the population. New antigenic variants that are not neutralised by this pre-existing immunity regularly emerge to replace previous lineages or antigenic clusters [19]. Detailed antigenic cartography of H3N2 viruses and molecular engineering of recombinant viruses revealed that a limited number of amino-acid positions located around the receptor binding site of the haemagglutinin protein are primarily involved in their antigenic drift [20]. Only one or two substitutions in this region of the protein typically differentiate newly emerging antigenic virus clusters. However, infection by a particular H3N2 variant was recently shown to boost immunity against most $\mathrm{H} 3 \mathrm{~N} 2$ antigenically distinct strains that circulated previously [21]. Such increasingly detailed understanding of influenza antigenic drift and antibody landscapes has strong potential to improve the strategic selection and engineering of seasonal influenza vaccine strains.

Influenza pandemic preparedness will continue to benefit from advances in influenza vaccine technology, as well as from improving global surveillance of animal and human influenza virus infections. One of the major obstacles currently faced by the global health community upon the emergence of a pandemic influenza virus is the delay between the identification of the new viral threat and the delivery of specific vaccines against it [22]. The prevailing manufacturing technology applied to influenza vaccine production today is based on techniques that are more than half a century old and require the propagation of vaccine viruses in embryonated chicken eggs, before inactivation and/or purification. This approach requires $>6$ months before the first vaccine doses can be used. Furthermore, the availability of fertilised chicken eggs limits the production capacity and rapid up-scaling at times of urgency. 
Novel technologies are currently being developed for the production of next-generation nonreplicating and live attenuated influenza vaccines, based on reverse genetics techniques and in vitro cell culture systems [22]. In addition, the use of adjuvants can improve the vaccines' protective efficacy, especially in individuals who are naïve towards a pandemic virus. The holy grail of influenza vaccinology is the development of a "universal influenza vaccine" or, perhaps more realistically, an influenza vaccine eliciting broader and longer-lasting immune responses against a range of influenza virus strains and subtypes. Advances in the identification of new correlates of protection, on the role of more conserved antigens and T-cell-mediated immune responses in protection are opening new avenues towards this goal [23]. Together, these modern approaches will undoubtedly improve the responsiveness of the global health community upon the emergence of a new pandemic.

Zoonotic influenza viruses that acquire the ability to transmit efficiently among humans via the air, through mutation, re-assortment or both, are at the origin of emerging influenza viruses with pandemic potential. The global surveillance of the diversity of circulating animal and a fortiori zoonotic influenza viruses can greatly improve our ability to anticipate which strains are more likely to evolve pandemic potential. Determining factors governing efficient airborne transmissibility of influenza viruses are the subject of thorough (and heavily debated) studies in the laboratory (for review see [24]). It has become clear that a handful of mutations suffice for an HPAIV H5N1 to acquire transmissibility among mammals. These have shed light on several genetic and phenotypic attributes associated with efficient airborne transmissibility, including haemagglutinin receptor binding specificity, glycosylation profile, thermostability and preferred $\mathrm{pH}$ for membrane fusion, as well as enhanced nuclear transport and viral transcription in mammalian cells [25-30]. Efficient replication in the human upper respiratory tract and in the soft palate may be phenotypic clues heralding the evolution of new pandemic viruses [31], yet their evolution remains difficult to forecast [32-34]. Furthermore, the evolution and adaptation of avian influenza viruses in mammalian species other than humans may provide unique opportunities to follow such gain of function in nature, further calling for dedicated surveillance programmes [35].

The race between influenza viruses that continually evolve towards inter- and subsequent intra-mammalian species transmission on the one hand, and humans aiming at gaining the ability to anticipate this evolution on the other, is at its fiercest. The threats will not cease to knock on our door; the stake lies in our ability to answer them.

\section{References}

$1 \quad$ Potter CW. A history of influenza. J Appl Microbiol 2001; 91: 572-579.

2 Hause BM, Collin EA, Liu R, et al. Characterization of a novel influenza virus in cattle and swine: proposal for a new genus in the Orthomyxoviridae family. MBio 2014; 5: e00031-14.

3 Taubenberger JK, Morens DM. 1918 influenza: the mother of all pandemics. Emerg Infect Dis 2006; 12: 15-22.

4 World Health Organization. Influenza (Seasonal). Fact sheet no. 211. Geneva, World Health Organization, 2014. Available from: www.who.int/mediacentre/factsheets/fs211/en/

5 Rimmelzwaan GF, Fouchier RA, Osterhaus AD. Age distribution of cases caused by different influenza viruses. Lancet Infect Dis 2013; 13: 646-647.

6 Fraaij PL, Heikkinen T. Seasonal influenza: the burden of disease in children. Vaccine 2011; 29: 7524-7528.

7 To KK, Chan JF, Chen $\mathrm{H}$, et al. The emergence of influenza A H7N9 in human beings 16 years after influenza A H5N1: a tale of two cities. Lancet Infect Dis 2013; 13: 809-821.

8 Toovey S. Influenza-associated central nervous system dysfunction: a literature review. Travel Med Infect Dis 2008; 6: $114-124$.

9 Nair H, Brooks WA, Katz M, et al. Global burden of respiratory infections due to seasonal influenza in young children: a systematic review and meta-analysis. Lancet 2011; 378: 1917-1930.

10 Dobson J, Whitley RJ, Pocock S, et al. Oseltamivir treatment for influenza in adults: a meta-analysis of randomised controlled trials. Lancet 2015; 385: 1729-1737.

11 Louie JK, Yang S, Samuel MC, et al. Neuraminidase inhibitors for critically ill children with influenza. Pediatrics 2013; 132: e1539-e1545.

12 Nuffield Dept of Primary Care Health Sciences. ALIC ${ }^{4}$ E: Antivirals for influenza Like Illness? An rCt of Clinical and Cost effectiveness in primary CarE. www.phc.ox.ac.uk/research/primary-care-clinical-trials-unit/infectious-diseases/ alic4e Date last accessed: February 7, 2016.

13 Platform for European Preparedness Against (Re-)emerging Epidemics, European Respiratory Society. PREPARE Education Virtual Learning Centre. Workpackage 4. http://prepare.ersnet.org/workpackages/workpackage-4.aspx Date last accessed: February 7, 2016.

14 van der Vries E, Stittelaar KJ, van Amerongen G, et al. Prolonged influenza virus shedding and emergence of antiviral resistance in immunocompromised patients and ferrets. PLoS Pathog 2013; 9: e1003343.

15 van der Vries E, Stelma FF, Boucher CA. Emergence of a multidrug-resistant pandemic influenza A (H1N1) virus. N Engl J Med 2010; 363: 1381-1382.

16 Beyer WE, McElhaney J, Smith DJ, et al. Cochrane re-arranged: support for policies to vaccinate elderly people against influenza. Vaccine 2013; 31: 6030-6033.

17 Russell CA, Jones TC, Barr IG, et al. The global circulation of seasonal influenza A (H3N2) viruses. Science 2008; 320: 340-346.

18 Bedford T, Riley S, Barr IG, et al. Global circulation patterns of seasonal influenza viruses vary with antigenic drift. Nature 2015; 523: 217-220. 
19 Smith DJ, Lapedes AS, de Jong JC, et al. Mapping the antigenic and genetic evolution of influenza virus Science 2004; 305: 371-376.

20 Koel BF, Burke DF, Bestebroer TM, et al. Substitutions near the receptor binding site determine major antigenic change during influenza virus evolution. Science 2013; 342: 976-979.

21 Fonville JM, Wilks SH, James SL, et al. Antibody landscapes after influenza virus infection or vaccination. Science 2014; 346: 996-1000.

22 Osterhaus A, Fouchier R, Rimmelzwaan G. Towards universal influenza vaccines? Philos Trans R Soc Lond B Biol Sci 2011; 366: 2766-2773.

23 Reperant LA, Rimmelzwaan GF, Osterhaus AD. Advances in influenza vaccination. F1000Prime Rep 2014; 6: 47.

24 Reperant LA, Kuiken T, Osterhaus AD. Adaptive pathways of zoonotic influenza viruses: from exposure to establishment in humans. Vaccine 2012; 30: 4419-4434.

25 Herfst S, Schrauwen EJ, Linster M, et al. Airborne transmission of influenza A/H5N1 virus between ferrets. Science 2012; 336: 1534-1541.

26 Imai $M$, Watanabe T, Hatta $M$, et al. Experimental adaptation of an influenza H5 HA confers respiratory droplet transmission to a reassortant $\mathrm{H} 5 \mathrm{HA} / \mathrm{H} 1 \mathrm{~N} 1$ virus in ferrets. Nature 2012; 486: 420-428.

27 Linster M, van Boheemen S, de Graaf M, et al. Identification, characterization, and natural selection of mutations driving airborne transmission of A/H5N1 virus. Cell 2014; 157: 329-339.

28 Gabriel G, Klingel K, Otte A, et al. Differential use of importin- $\alpha$ isoforms governs cell tropism and host adaptation of influenza virus. Nat Commun 2011; 2: 156.

29 Tumpey TM, Maines TR, Van Hoeven N, et al. A two-amino acid change in the hemagglutinin of the 1918 influenza virus abolishes transmission. Science 2007; 315: 655-659.

30 Sutton TC, Finch $\mathrm{C}$, Shao $\mathrm{H}$, et al. Airborne transmission of highly pathogenic H7N1 influenza virus in ferrets. J Virol 2014; 88: 6623-6635.

31 Lakdawala SS, Jayaraman A, Halpin RA, et al. The soft palate is an important site of adaptation for transmissible influenza viruses. Nature 2015; 526: 122-125.

32 Russell CA, Fonville JM, Brown AE, et al. The potential for respiratory droplet-transmissible A/H5N1 influenza virus to evolve in a mammalian host. Science 2012; 336: 1541-1547.

33 Reperant LA, Kuiken T, Grenfell BT, et al. The immune response and within-host emergence of pandemic influenza virus. Lancet 2014; 384: 2077-2081.

34 Reperant LA, Grenfell BT, Osterhaus AD. Quantifying the risk of pandemic influenza virus evolution by mutation and re-assortment. Vaccine 2015; 33: 6955-6966.

35 Bodewes R, Bestebroer TM, van der Vries E, et al. Avian influenza A(H10N7) virus-associated mass deaths among harbor seals. Emerg Infect Dis 2015; 21: 720-722. 